\title{
Spontaneous Root-Nodule Formation in the Model Legume Lotus japonicus: A Novel Class of Mutants Nodulates in the Absence of Rhizobia
}

\author{
Leïla Tirichine, ${ }^{1}$ Euan K. James, ${ }^{2}$ Niels Sandal, ${ }^{1}$ and Jens Stougaard1 \\ ${ }^{1}$ Laboratory of Gene Expression, Department of Molecular Biology, University of Aarhus, Gustav Wieds Vej 10, \\ DK-8000 C Aarhus, Denmark; ${ }^{2}$ CHIPs, MSI/WTB Complex, SLS, University of Dundee, Dundee DD1 5EH, U.K.
}

Submitted 28 November 2005. Accepted 2 January 2006.

Root-nodule development in legumes is an inducible developmental process initially triggered by perception of lipochitin-oligosaccharide signals secreted by the bacterial microsymbiont. In nature, rhizobial colonization and invasion of the legume root is therefore a prerequisite for formation of nitrogen-fixing root nodules. Here, we report isolation and characterization of chemically induced spontaneously nodulating mutants in a model legume amenable to molecular genetics. Six mutant lines of Lotus japonicus were identified in a screen for spontaneous nodule development under axenic conditions, i.e., in the absence of rhizobia. Spontaneous nodules do not contain rhizobia, bacteroids, or infection threads. Phenotypically, they resemble ineffective white nodules formed by some bacterial mutants on wild-type plants or certain plant mutants inoculated with wild-type Mesorhizobium loti. Spontaneous nodules formed on mutant lines show the ontogeny and characteristic histological features described for rhizobiainduced nodules on wild-type plants. Physiological responses to nitrate and ethylene are also maintained, as elevated levels inhibit spontaneous nodulation. Activation of the nodule developmental program in spontaneous nodules was shown for the early nodulin genes Enod2 and Nin, which are both upregulated in spontaneous nodules as well as in rhizobial nodules. Both monogenic recessive and dominant spontaneous nodule formation (snf) mutations were isolated in this mutant screen, and map positions were determined for three loci. We suggest that future molecular characterization of these mutants will identify key plant determinants involved in regulating nodulation and provide new insight into plant organ development.

The Leguminosae is one of the largest plant families and includes agronomically important species such as soybean, beans, pea, peanuts, and forage legumes. One of the agricultural and ecological advantages of legumes is their ability to establish symbiosis with nitrogen-fixing bacteria, known collectively as rhizobia. This access to combined nitrogen via its microbial partner reduces the need for expensive nitrogen fertilizers in legume crops and is an important feature of sustainable agriculture. As alternative, legumes can also utilize the normal sources of nitrogen available in the soil by adjusting nitrogen metabolism accordingly. High levels of nitrate will, for example, suppress nodule formation and shift nitrogen me-

Corresponding author: J. Stougaard; Telephone: +00 45894250 11; Fax: +00 45871231 78; E-mail: stougaard@mb.au.dk tabolism towards growth on nitrate (Wopereis et al. 2000). Root-nodule formation is thus a dispensable developmental process and an example of induced postembryogenic organogenesis in plants.

Rhizobia-legume symbiosis involves a set of plant and bacterial genes interacting in a complex process leading to initiation and development of root nodules. Organogenesis is triggered by the rhizobial microsymbiont, but the legume host plant encodes the developmental program responsible for building the nodule tissues and for regulating the process. Lipochitin-oligosaccharides (Nod factors) synthesized and secreted by rhizobia are major signal molecules triggering nodule developmental processes in legume host plants (Lerouge et al. 1990; Spaink 2000). Purified Nod factors function as mitogens or morphogens that, applied to roots in nano- to picomolar concentrations, induce formation of nodule structures on compatible legumes (Demont-Caulet et al. 1999; Gage 2004; Niwa et al. 2001). In Lotus japonicus, two genes, Nfrl and Nfr5, encoding receptor kinases with LysM domains were shown to be involved in Nod-factor perception (Madsen et al. 2003; Radutoiu et al. 2003) and, together with a leucine-rich repeat receptor-kinase gene, SymRK (Stracke et al. 2002), required for initiating the signal transduction leading to nodule development. In pea and Medicago spp., the SYM10, LYK3/LYK4 and SYM19, NORK/DMI2 genes serve similar functions (Endre et al. 2002; Limpens et al. 2003; Madsen et al. 2003). Oldroyd and Downie (2004) recently reviewed literature on early signal transduction events.

To establish symbiosis, rhizobia are endocytosed into single plant cells in which they are surrounded by a peribacteroid membrane in the symbiosomes. In Lotus japonicus, infection occurs via infection threads that pass through root hairs into the root cortex and distribute bacteria to cells, which become infected symbiosome-containing nitrogen-fixing cells (Karas et al. 2005; van Spronsen et al. 2001). Legumes are particularly susceptible to invasion in a root zone located behind the root tip, in which root hairs are still growing and competent for invasion. In response to attached bacteria, root hairs deform and curl, setting up a pocket that provides a site for infectionthread initiation (Geurts et al. 2005). Infection threads are plant-derived structures originating from plasma membrane invagination accompanied by external deposition of cell wall material. In advance of the inward progressing intracellular thread, root cortical cells dedifferentiate and reenter the cell cycle (Foucher and Kondorosi 2000). In Lotus japonicus, cell divisions in the third cortical cell layer are most frequently initiating the primordium (van Spronson et al. 2001). Later in the process, pattern formation and cell differentiation specify tissue 
and cell types, including the infected cells that contain rhizobia. In the mature functional nodule, peripheral vascular bundles are connected to the root vasculature and the main tissues and cell types can be distinguished (Pawlowski and Bisseling 1996). Presence of bacterial microsymbionts is indispensable for formation of functional nitrogen-fixing root nodules, but previous work reported the capacity of certain varieties of alfalfa and clover to develop empty nodules in the absence of rhizobia (Blauenfeldt et al. 1994; Joshi et al. 1991, 1993; Truchet et al. 1989). These varieties presumably carry allele variants of regulatory genes or genes mediating root-nodule initiation, but the genetic complexity of tetraploid alfalfa and white clover prevented further studies.

Molecular characterization of spontaneous nodulation mutants, uncoupling bacterial signaling from nodule organogenesis, would greatly facilitate genetic dissection of nodule development. Considering the importance of this category of mutants, we performed a large-scale screening for spontaneous nodulation in the model legume Lotus japonicus, which has all the features suitable for genetic studies. It is diploid, has a small genome, and a considerable amount of resources are available in Lotus japonicus, including a genome sequencing program (Asamizu et al. 2003; Handberg and Stougaard 1992; Kawaguchi et al. 2001; Pedrosa et al. 2002; Sandal et al. 2002). Here, we report isolation of six genetically stable spontaneous rootnodule formation mutant lines and initial phenotypic characterization of four lines, snfl, snf2-1, snf2-2, and snf4.

\section{RESULTS}

\section{Identification of spontaneous nodulation mutants of Lotus japonicus.}

Assuming that relatively few mutations would cause spontaneous nodulation and mutants would be rare, we screened plants from an ethyl methyl sulfonate (EMS)-mutagenized population already known to produce mutants (Perry et al. 2003). The screen was done on 5-week-old plants grown at high density under rhizobia-free axenic conditions and in the absence of nitrogen-containing nutrients. Approximately 500,000 M3 seeds originating from 2,400 M2 pools, each containing seeds from two to ten M2 plants, were surface-sterilized, germinated, and left undisturbed in autoclaved growth support until the screening (discussed below). In the primary screening, putative mutants displaying nodule-like structures were found in 86 out of 2,400 seed pools. Each of these putative mutants was rescued by transfer to nitrogen-containing nutrients and was grown for seed production. Progeny of each self-fertilized plant were subsequently screened for spontaneous nodulation in order to confirm phenotype and inheritance of spontaneous nodule development. $\mathrm{Mu}-$ tants from six pools reproduced the mutant phenotype demonstrating stability of the spontaneous nodule formation (snf) phenotype. Progeny of the 80 remaining putative mutant plants did not show any nodule-like structures. These 80 plants were all rescued from pools in which only one putative mutant plant with one nodule-like structure was found. The six genetically stable snf mutants were isolated from pools in which one or more plants developed more than one nodule-like structure or in pools in which more than one plant developing one spontaneous nodule were found. Mutations causing spontaneous nodulation were relatively rare since only $0.25 \%$ of the 2,400 EMS M2 lines gave rise to spontaneous nodulation mutants.

Spontaneously nodulating mutant plants grown under axenic nitrogen-deficient conditions are nitrogen-starved, indicated by anthocyanin accumulation in the stem, light green leaves, and poor growth of the aerial part of the plants (Fig. 1A). Nodules are white, round-shaped, and distributed on a normal-looking root system (Fig. 1A and C). snf nodules are empty.

Spontaneous nodulation mutants were found using growth conditions in which considerable effort was invested in excluding rhizobia. In order to confirm that spontaneous nodule formation was a plant mutant phenotype and not a result of, for example, delayed infection from accidentally contaminating rhizobia, the bacterial content of nodules was examined. Five spontaneous nodules from five different plants of each $s n f$ line were surface-sterilized, crushed, and plated on standard rhizobial yeast-mannitol agar (YMA) medium. No rhizobia or other bacteria were recovered from these 30 nodules. Further evidence for the absence of rhizobia was obtained by crushing three non-surface sterilized snf nodules from individual plants of each snf line and inoculating wild-type Lotus plants using the suspensions. None of the plants inoculated with these 18 suspensions developed nodules. These results show that snf nodules were empty and free of any bacteria capable of nodulating Lotus plants (data not shown).

\section{Spontaneous nodules are genuine nodules.}

Light microscopy and scanning electron microscopy was used to describe the histology of spontaneous nodules and to ascertain that spontaneous nodules developing on mutant lines were indeed empty nodules and not aberrant root swellings. The representative pictures and sections shown here are mainly from $s n f 2-1$ and $s n f 2-2$ mutants, but all four snf mutant lines examined for phenotype and nodule ontogeny showed similar results (data not shown). Thin sections show that $s n f 2-1$ nodules have two peripheral vascular bundles like wild-type rhizobial nodules (Fig. 1D and E). Peripheral vascular bundles are a characteristic feature distinguishing root nodules from lateral roots, which have one central vascular bundle. The globular shape and attachment in the root cortex is a characteristic result of determinate nodule development and very different from the elongated cell file arrangement typical for lateral roots that grow through the cortex. Some of the larger spontaneous nodules have cells of a size reminiscent of infected cells characteristic for rhizobia-induced nodules (Fig. 1E). This may indicate that endoreduplication occurs in a later stage of spontaneous nodule development. Staining of $s n f 2-2$ nodules with potassium iodide revealed a massive presence of starch granules (Figs. 1E, 2B and C), as observed in nonfixing plant mutants and wild-type plants inoculated with Fix $^{-}$rhizobial mutants (Tansengco et al. 2003). Electron microscopy confirmed the absence of bacteria, infection threads, and bacteroids in snf nodules. As for the light microscopy, electron microscopy showed no ultrastructural differences between wild-type and snf nodules except for absence of bacteroids and presence of starch granules (Fig. 2A, B, and C). Cells in snf nodules show a dense cytoplasm with many mitochondria and lipid bodies. Sections of vascular tissue show xylem vessels with many branched wall ingrowths (Fig. 2D), but transfer cells as reported for alfalfa spontaneously nodulating mutants (Joshi et al. 1993) were not observed. Altogether, these results show that the snf nodules are, indeed, genuine nodules with histological features characteristic for empty root nodules.

\section{Root cortical-cell division pattern in $s n f$ mutants.}

Activity of Nin promoter- $\beta$-glucuronidase (GUS) fusion marks root-nodule primordial cells of transgenic wild-type plants (Fig. 1F). In order to trace cell divisions in the early phase of spontaneous nodule formation, the same Nin promoterGUS fusion construct was crossed into snf2-2 mutant background. As shown in Figure 1G, Nin-GUS activity was also found in spontaneous nodule primordia and cortical-cell division foci initiating nodule primordia in $s n f 2-2$ roots. Lotus root 
nodules are initiated by cortical-cell division. To trace cell division patterns in snf mutants, nodules at 15 days postgermination were sectioned transversally. Serial thin sections $(4 \mu \mathrm{m})$ were inspected, and cortical-cell divisions forming the nodule primordia were found developing in front of xylem poles of the root (Fig. 1I) as in the wild-type nodule (Fig. 1H). Serial sections shown in Figure 1I and J clearly indicate Nin-GUS activity in nodule primordia.

\section{Enod 2 and Nin gene expression are upregulated in snf mutants.}

One of the features distinguishing rhizobia-induced root nodules from root tissue is specific or highly upregulated expression of a gene set encoding the nodulin proteins involved in nodule organogenesis or required for root-nodule function. Two nodulin genes, Enod 2 and Nin, expressed at different stages of wild-type nodule development were chosen to compare nodulin gene expression in spontaneous nodules of snfl, snf2-1, snf2-2, snf4, and wild-type nodules formed after inoculation with $M$. loti. The Enod 2 gene encodes a hydroxyprolinerich protein expressed in nodule parenchyma and serves as a marker for organ development and differentiation (Niwa et al. 2001; van de Wiel et al. 1990). Nin encodes a putative transcriptional regulator required for infection-thread formation and inception of nodule primordia (Schauser et al. 1999). Nin is first expressed a few hours after inoculation with M. loti, and expression continues into mature nodules serving as a marker for both initiation and development. Both Enod2 and Nin gene expression were upregulated to comparable levels in snf nodules and wild-type rhizobial nodules. Compared with uninoculated roots of wild type, the upregulation observed was 10 -fold or more (Fig. 3). In spontaneous nodules of alfalfa, ENOD2 was expressed in the same tissue as in wild-type nodules (Hirsch et al. 1992). In M. loti-inoculated wild-type plants Nin-GUS expression is first detected in a zone overlapping or marking the invasion zone in which legumes are most susceptible to rhizobial infection (Radutoiu et al. 2003). Later in nodule development, Nin is expressed in nodule primordia and nodules (Radutoiu et al. 2003; Schauser et al. 1999). A comparable expression pattern illustrated in a snf2-2 mutant back-

Fig. 1. Phenotype of spontaneous nodulation mutants. A, To the left, a Lotus japonicus wild-type plant inoculated with Mesorhizobium loti. Symbiotic nodules marked with arrows. To the right, snf 2 mutant with spontaneous nodules (arrowheads), grown without nitrogen and in the absence of rhizobia. Picture taken 5 weeks postgermination. B, Symbiotic root nodules induced on wild-type Lotus japonicus ecotype Gifu after inoculation with $M$. loti. Bar $=1,000 \mu \mathrm{m}$. C, Spontaneous nodules formed on snf1, 5 weeks postgermination. Bar $=500 \mu \mathrm{m}$. D, Longitudinal thin section $(4 \mu \mathrm{m})$ of $s n f 2-1$ nodule. Bar $=100 \mu \mathrm{m}$. E, Longitudinal thin section $(4$ $\mu \mathrm{m})$ of $\operatorname{snf} 2-2$ mutant after staining with potassium iodide. White arrowheads show accumulation of starch granules. Vascular strands are indicated by black arrowheads in both D and E. Bar $=50 \mu \mathrm{m}$. F, Entire root of promoter Nin- $\beta$-glucuronidase (GUS) transformed line of wild-type Lotus japonicus inoculated with $M$. loti. G, Spontaneous nodules on F1 plant generated by a cross between $s n f 2-2$ and Nin-GUS lines. GUS activity was detected only in nodule primordia. Bar $=5 \mathrm{~mm}$. H, I, and $\mathbf{J}$, Transversal thin sections through a rhizobial-induced nodule primordium on wild-type (H) and spontaneous snf2-2 nodule primordia carrying the promoter NinGUS construct (I and J). Nodule primordia develops in front of the xylem pole shown by black arrows ( $\mathrm{H}$ and $\mathrm{I})$. Stars indicate cell divisions. Only dividing cortical cells, in front of the xylem pole, stain blue. Bar $=50 \mu \mathrm{m}$. $\mathbf{K}$, Rhizobial-induced (white arrowheads) and spontaneous nodules (black arrowheads) developing on snf2-1 mutants 5 weeks postgermination. The plants were inoculated with $M$. loti NZP2235. Bar $=5 \mathrm{~mm}$. L, Root of snf2-2 mutant showing arbuscule development of mycorrhiza (white stars). Roots cleared and stained with the ink vinegar procedure. White arrows show fungal hyphae growth. Bar $=5 \mu \mathrm{m}$. ground (Fig. 1G, I, and J) and lack of ectopic expression of Nin-Gus suggests that the organogenic program is not constitutively activated in snf plants. Apparently, other conditions are required, and spontaneous nodulation is still integrated into the overall developmental control, regulating the wild-type noduledevelopmental process. Altogether, the gene-expression pattern detected in spontaneous nodules reflects the expression pattern in rhizobia-induced nodules and is clearly different from the root expression pattern.
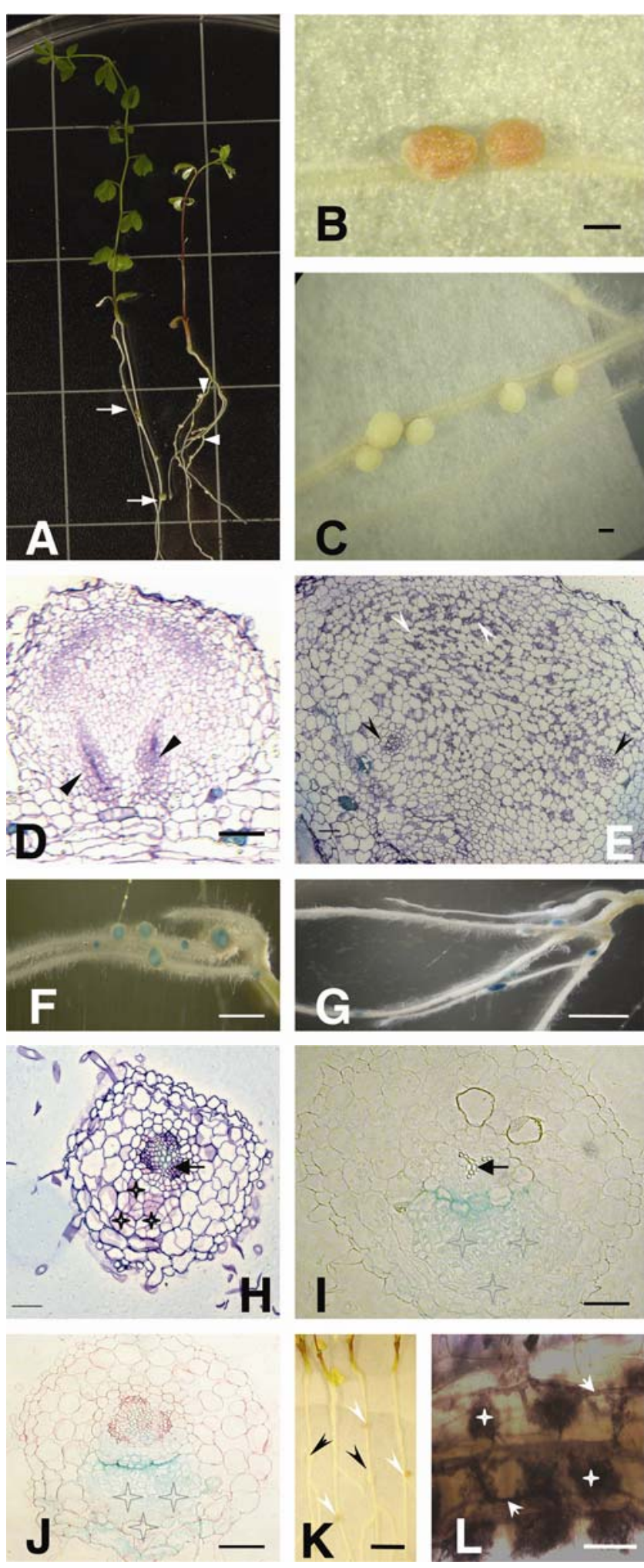


\section{Nodulation is regulated in snf mutants.}

In wild-type plants, nodulation by rhizobia is regulated by the legume host (Caetano-Anolles, and Gresshoff 1991; Krusell et al. 2002; Searle et al. 2003). In Lotus japonicus, a microscopical analysis of bacterial invasion events accompanied by nodule primordia demonstrated that most infection threads were arrested and did not give rise to a nodule (Wopereis et al. 2000). Only about $10 \%$ of infections were directly associated with a root-nodule primordium. The hypernodulating harl mutants of Lotus japonicus were less restrictive, and 40 to $50 \%$ of infection threads were associated with a primordium, demonstrating genetic control of infection events as well as nodule numbers (Krusell et al. 2002). Since nodule primordium formation is completely uncoupled from bacterial infection events in snf mutants, we have monitored the nodulation of $s n f 1, \operatorname{snf} 2-1, \operatorname{snf2-2}$, and snf4 mutants in the presence and absence of rhizobia. On agar plates, the first rhizobia-induced nodules on wild-type plants are visible under a stereomicroscope approximately 7 days postgermination and inoculation (Fig. 4A) and reach a plateau of 4.5 nodules after 40 days. snf mutants develop normal nodules after inoculation with $M$. loti, and these nodules are visible 7 days postinoculation, as in wild-type plants. Like wild type, snfl mutants develop 4.5 nodules, while snf2-1 and snf2-2 mutants develop an average of three nodules. In snf4, which develops only one nodule in the absence of $M$. loti, an average of four nodules per plant develop after inoculation with $M$. loti. In general, rhizobia-induced nodule numbers in snfl and snf4 mutants reaches the plateau a week earlier than in wild-type plants and nodule development appears to progress slightly faster in snf1 mutants. Interestingly, rhizobia-induced nodules on snf mutants do not completely suppress spontaneous nodulation (Fig. $1 \mathrm{~K}$ ), as spontaneous and rhizobial nodules are occasionally observed on the same root. Figure $1 \mathrm{~K}$ shows results from a nodulation test on plates. Parallel experiments in jars confirmed the plate results (data not shown).
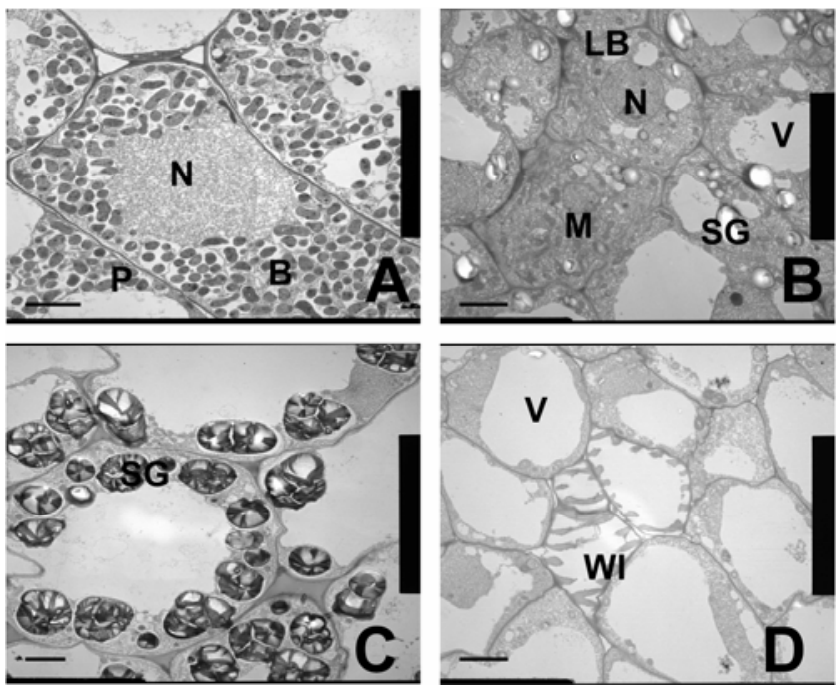

Fig. 2. Electron micrographs. A, Wild-type Mesorhizobium loti-induced root nodule showing infected cells full of bacteroids and $\mathbf{B}$, spontaneous snf1 nodule 5 weeks after germination showing noninfected cells without any bacteria. Note the prominent nucleus, an electron-dense cytoplasm with mitochondria, starch grains, and lipid bodies. C, Cells in a snfl nodule containing a large number of starch grains. D, Vascular tissue in 5week-old spontaneous nodule from the snf1 mutant showing xylem vessels and xylem parenchyma with wall ingrowth. $\mathrm{N}=$ nucleus, $\mathrm{M}=$ mitochondria, $\mathrm{V}=$ vacuole, $\mathrm{SG}=$ starch grain, $\mathrm{B}=$ bacteroid, $\mathrm{P}=$ plasma membrane, $\mathrm{LB}=$ lipid body, and $\mathrm{WI}=$ wall ingrowth. Bars $=4 \mu \mathrm{m}$.
Spontaneous nodulation in the snfl, snf2-1, and snf2-2 is observed 10 days postgermination and $s n f 2-1$ and $s n f 2-2$ reach a plateau 30 days postgermination (Fig. 4B). With an average of two nodules, the nodulation frequency of snf2-1 and snf2-2 mutants is similar. The snfl mutant develops around eight nodules, and the nodule number stabilizes later, approximately 66 days postinoculation (Fig. 4B). Variation in penetrance between the mutations could explain this difference.

\section{Nitrogen inhibits spontaneous nodulation.}

Rhizobia-induced nodulation of Lotus japonicus is nitrogensensitive, and nitrogen-limited conditions promote root-nodule formation. In the presence of high concentrations of nitrate, the plant shifts its nitrogen metabolism towards nitrate assimilation and nodulation is suppressed (Wopereis et al. 2000). Under these conditions, nodule organogenesis is arrested, resulting in a reduced number of nodules and development of prematurely arrested nodule structures rather than functional

\section{Nin and Enod2 expression in the snf mutants}

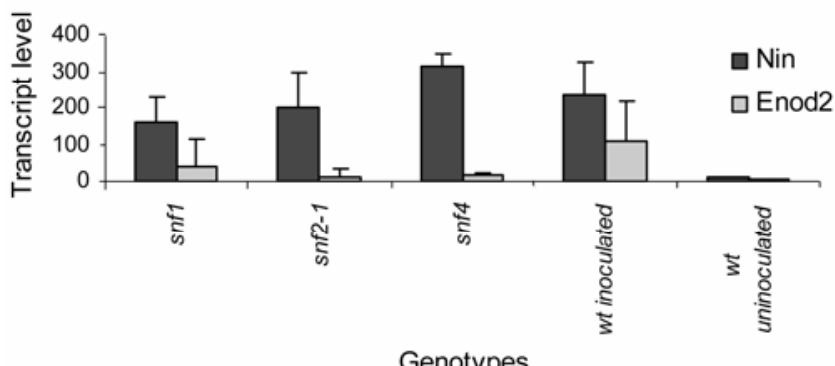

Fig. 3. Expression of Nin and Enod2 nodulin genes in wild-type and snf nodules. RNA was extracted from mutants 5 weeks postgermination and relative transcript levels were determined by quantitative polymerase chain reaction. Uninoculated wild-type roots are used as a reference at time zero. ATPase gene was used as an internal control and relative values normalized to the uninoculated root are shown, and $95 \%$ confidence intervals are indicated.

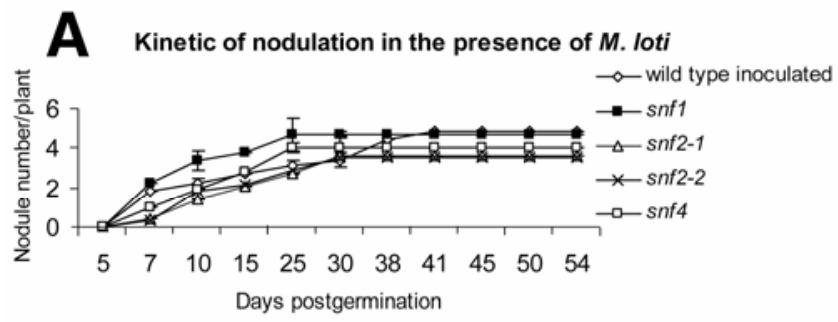

B Kinetic of snf mutants nodulation in the absence of $M$. loti

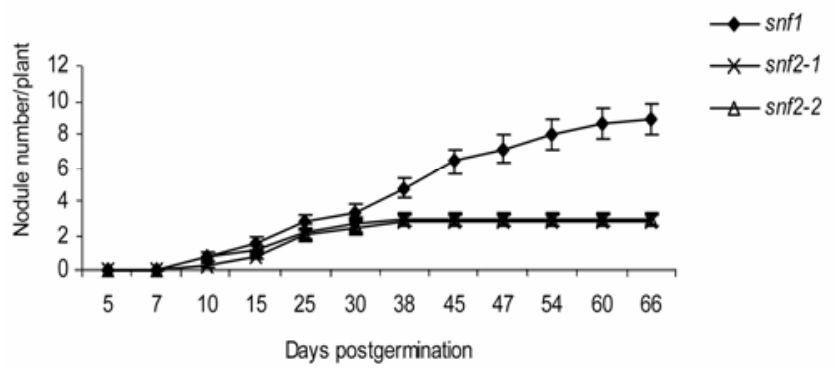

Fig. 4. Nodulation timecourse for snf mutants. A, Nodulation in the presence of Mesorhizobium loti. B, Spontaneous nodulation on snf mutants in the absence of rhizobia. Timepoints beyond 66 days were also monitored. Approximately 80 plants for each mutant were scored at regular intervals for nodules appearance, and $95 \%$ confidence intervals are indicated. 
nodules. To characterize spontaneous nodulation further, we investigated whether spontaneous nodulation is regulated by the mechanism controlling rhizobial nodule development in the presence of nitrate. To examine this aspect of nitrogen regulation, snf mutant seedlings were grown axenically on plates containing different concentrations of potassium nitrate. Nodule number decreases significantly at $5 \mathrm{mM} \mathrm{KNO}_{3}$ (Fig. $5 \mathrm{~A}$ ), especially in snfl mutants. At $20 \mathrm{mM}$, no fully developed nodules were observed in snf mutants. Only a few bumps were formed. For comparison, results from nitrate suppression of $M$. loti-induced nodules on wild-type plants are included in Figure 5A.

\section{Ethylene inhibits spontaneous nodulation.}

Ethylene regulates rhizobia-induced nodulation negatively (Goodlass and Smith 1979; Peters and Crist-Esters 1989). Although not all legumes respond similarly (Schmidt et al. 1999), addition of exogenous ethylene to nodulating pea, al-
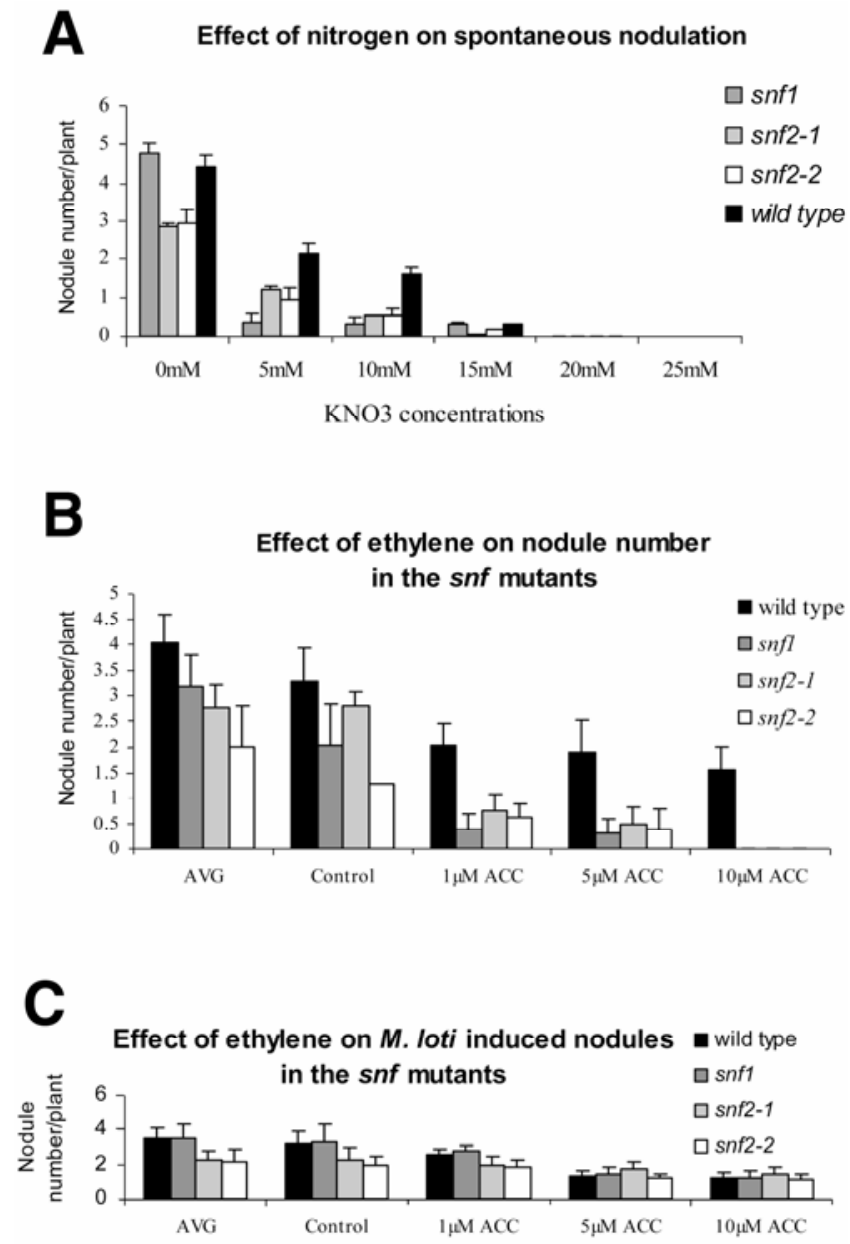

Fig. 5. Suppression of nodulation. A, Effect of increasing nitrate concentration on Mesorhizobium loti-induced nodulation of wild type and spontaneous nodulation of snf mutants. B, Effect of increasing concentration of the ethylene precursor 1-aminocyclopropane-1-carboxylic acid (ACC) on $M$. loti-induced nodulation of wild type and spontaneous nodulation of snf mutants. C, Effect of increasing concentration of the ethylene precursor ACC on $M$. loti-induced nodulation of wild type and snf mutants. The effect of amino ethoxyvinyl glycine (AVG; $1.27 \mathrm{nM}$ ) on $M$. loti-induced nodulation of wild type is shown in both $\mathrm{B}$ and $\mathrm{C}$, while the effect on spontaneous and rhizobial nodulation of snf mutants is shown in B or $\mathrm{C}$, respectively. The seedlings of the snf mutants were grown on solid B and D nutrient plates supplied with different concentrations of ACC or AVG as indicated. Nodule number was assessed 5 weeks postgermination, and 95\% confidence intervals are indicated. falfa, and Lotus plants reduces the frequency of nodule primordium formation (Heidstra et al. 1997; Nukui et al. 2000; Oldroyd et al. 2001). Endogenously produced ethylene also inhibits nodulation, and treatment of certain legumes with amino ethoxyvinyl glycine (AVG), an inhibitor of 1-aminocyclopropane-1-carboxylic acid (ACC) synthase, increases nodule numbers. To compare nodule numbers in the presence and absence of endogenous ethylene biosynthesis, seedlings were grown in the presence of $1.27 \mathrm{nM}$ AVG. This concentration is known to increase rhizobial nodulation in Lotus japonicus (van Spronsen et al. 2001), although a significant

Table 1. Segregation analysis of the $s n f$ mutants ${ }^{\mathrm{a}}$

\begin{tabular}{lcc}
\hline Mutant & ${\text { Segregation in F2 } \text { population }^{\mathbf{b}}}$ & $\chi^{\mathbf{2}}$ \\
\hline snf1 & $289 / 69$ & 1.74 \\
snf2-1 & $99 / 245$ & 0.75 \\
snf2-2 & $45 / 142$ & 0.42 \\
snf4 $^{c}$ & $26 / 6$ & 2.08 \\
\hline
\end{tabular}

a The qui square analysis was used to determine whether the segregation ratios are different from $3: 1$ at $P=0.05, \chi^{2}=3.84$.

${ }^{\mathrm{b}}$ Values = wild type/snf mutant. The analyzed $\mathrm{F} 2$ populations come from a cross between $L$. japonicus Miyakojima and every snf mutant.

c The segregation of the snf4 mutant was scored 9 weeks postgermination, while for the remaining snf mutants, the segregation was scored 5 weeks postgermination.

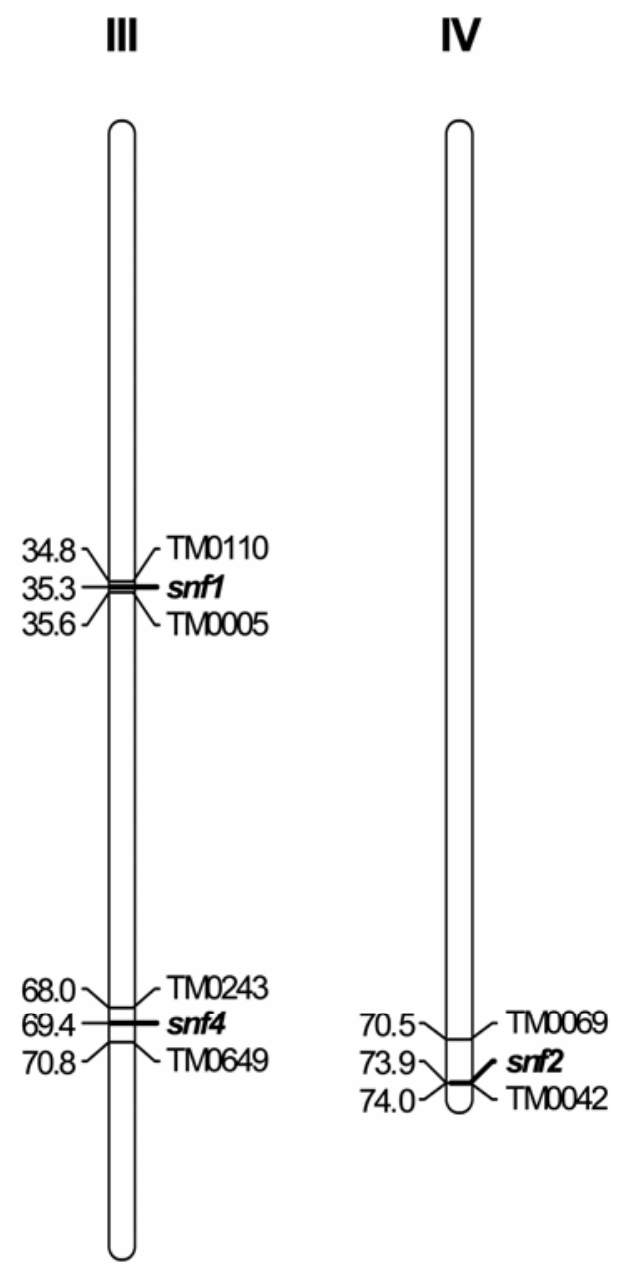

Fig. 6. Positions of snf loci on the genetic map of Lotus japonicus. The flanking microsatellite markers (Hayashi et al. 2001; Kato et al. 2003) and the distances in centimorgans are indicated. The mapping populations were generated from a cross between $L$. japonicus Miyakojima and each of the snf1, snf2, and snf4 mutants present in a L. japonicus Gifu genetic background. 
increase was not observed under our conditions (Fig. 5B and C). To determine whether ethylene has a regulatory effect on spontaneous nodulation of snf mutants, we added different concentrations of ACC, a precursor of ethylene, in nodulation plate tests. The nodule number per plant was scored 5 weeks postgermination. Nodule numbers in snf mutants and M. lotiinoculated wild type decline with increasing concentrations of the ethylene precursor ACC. Spontaneous nodulation was totally inhibited at $10 \mu \mathrm{M}$ ACC, while nodulation of wildtype plants was reduced to $50 \%$. In order to determine if this was a real difference between spontaneously formed nodules and rhizobial-induced nodules, we determined the rhizobial nodulation frequency of snf mutants in the presence of ACC. The results show that $M$. loti-induced nodulation of snf mutants in the presence of ACC was not totally inhibited. Nodule number decreases with increasing concentrations of ACC. At $10 \mu \mathrm{M}$ ACC, snf mutants are still developing rhizobial nodules (Fig. 5C). It seems that spontaneous nodulation is more sensitive to ethylene than rhizobial nodulation. The root system of both the snf mutants and wild-type plants becomes shorter and thicker with increasing concentrations of ACC (data not shown).

\section{snf mutants are colonized by mycorrhiza.}

Lotus japonicus, like other legumes, can enter into symbiosis with mycorrhizal fungi belonging to division Glomeromycota. Roots are colonized by fungal hyphae, and vesicular arbuscular mycorrhizal symbiosis is established. Some nonnodulating Lotus plant mutants impaired early in rhizobial symbiosis were also impaired in mycorrhizal colonization, and genetic analysis defined seven loci constituting a common signal transduction pathway (Kistner et al. 2005). A change in mycorrhization of snf mutant plants was therefore a possibility. To resolve this question, colonization by Glomus intraradices was investigated. The snf1, snf2-1, snf2-2, and snf4 mutants appeared to have wild-type-like mycorrhization. Observed by light microscopy, appressoria development, hyphae penetration and branching (Fig. 1L) seemed normal. Apparently snf mutants are neither less susceptible nor hyperinfected by mycorrhiza. The infection is as widely spread as observed in wild-type control plants (data not shown).

\section{Genetic analysis.}

For snf1, snf2-1, snf2-2, and snf4 mutants, several crosses with the Miyakojima ecotype were made to assess the inheritance of the spontaneous nodulation phenotype (Table 1). The F2 progeny from snfl crosses segregate 69 snf mutants and 289 wild types, indicating monogenic recessive inheritance of snf1. For snf2-1 and snf2-2, segregation approximates 1:3 wild type to snf mutants (Table 1), indicating that they are inherited as monogenic dominant loci. Scoring of the snf4 phenotype was possible 8 to 10 weeks postgermination. snf4 segregates as a single recessive gene (Table 1). Two independent diallelic crosses show that $s n f 2-1$ and $s n f 2-2$ are either allelic or very closely linked and, therefore, designated as snf2-1 and snf2-2 alleles. More than 100 F2 individuals of each cross were screened in the absence of rhizobia, and none of the crosses showed segregation of wild-type plants. Two independent crosses show that snfl and snf4 define two different loci. Furthermore, genetic mapping of $s n f 1, s n f 2$, and snf4 to three different positions on chromosome III and IV confirm that they are independent loci (Fig. 6). Two spontaneously nodulating mutant lines were isolated recently, and segregating populations are not yet available. Scoring of spontaneous nodulation mutants and wild-type plants segregating in self-progeny of heterozygous plants indicate that both are inherited as dominant loci (data not shown).

\section{DISCUSSION}

Six spontaneous nodulation mutants of Lotus japonicus developing root nodules in the absence of Mesorhizobium loti were isolated from an EMS-mutagenized population. Two mutant loci (snfl and snf4) were monogenic recessive, while one locus represented by two alleles (snf2- 1 and $s n f 2-2$ ) was monogenic dominant. Genetic mapping in $\mathrm{F} 2$ populations positioned snfl and snf4, respectively, at 35.3 and $69.4 \mathrm{cM}$ of linkage group III, while snf 2 maps at $73.9 \mathrm{cM}$ of linkage group IV. Genetic markers flanking these positions segregate without distortion in both the general F2 mapping population (Hayashi et al. 2001; Kato et al. 2003) and the derived recombinant inbred lines (Sandal et al. 2005), strongly supporting our assignment of dominance from segregation in F2 mapping populations.

Several lines of evidence support the origin and identity of spontaneous nodules as genuine root nodules. Initial plating and plant inoculation of extracts from crushed spontaneous nodules did not recover rhizobia, confirming that snf nodules were empty and formed without perceiving rhizobial signals. In line with this result, subsequent light and electron microscopy studies did not detect the presence of infected cells, bacteria, bacteroids, or infection threads. Inspection of root sections show that cell divisions initiating spontaneous nodules occur in the root cortex in positions similar to cell divisions, giving rise to nodules induced by rhizobia (van Spronsen et al. 2001). This shared cortical origin of spontaneous nodules and rhizobial-induced nodules suggests a common ontogeny. Development of two peripheral vascular bundles characteristic for rhizobial-induced root nodules and localization of snf nodules in front of protoxylem poles, where nodule primordia predominantly develop (Libbenga and Harkes 1973; Heidstra et al. 1997), lend further support to this interpretation. Similar to ineffective $\left(\mathrm{Fix}^{-}\right)$nodules developing on plant mutants arrested in nodule organogenesis and in nodules induced by bacterial mutants, the snf nodules accumulate starch granules (Kawaguchi et al. 2002; Leigh et al. 1985). Electron microscopy shows that snf nodules exhibit normal ultrastructure. The vascular tissue contains xylem and phloem vessels but no associated transfer cells as reported for alfalfa were observed (Joshi et al. 1991). This is in accordance with previous reports suggesting that transfer cells are present only in Vicieae and Trifolieae tribes (Sprent and Embrapa 1980). At the level of gene regulation, induction of Nin and Enod2 gene expression in spontaneous nodules indicate activation of the nodule developmental program required for nodule inception and organogenesis. We conclude that spontaneous nodules on Lotus snf mutants are caused by a plant mutation and the observed spontaneous nodules are formed independently of rhizobia and other bacteria capable of inducing nodule structures. Altogether, our results show that spontaneous nodules in Lotus japonicus are genuine nodules presenting the histological features of $M$. loti-induced nodules and not the result of an aberrant development of lateral structures.

Nodule-like structures resulting from treatment with hormones or hormone antagonists were previously reported in legumes (Allen et al. 1953; Hirsch and Fang 1994; Hirsch et al. 1989). Unlike the snf nodules in Lotus japonicus, these nodule-like structures show disorganized cellular structure, even though in some cases, they originate from cortical-cell divisions. In contrast to snf nodules showing two peripheral vascular bundles, they are connected to the root by a central vascular bundle like lateral roots (Hirsch et al. 1989). More similar to snf nodules are the empty nodules formed by rhizobial mutants impaired in synthesis of lipopolysaccharides, exoploysaccharides, and $\beta$-glucan (Finan et al. 1985; Fraysse et al. 2003; Gray et al. 1992). As in snf nodules, neither infec- 
tion threads nor intracellular bacteria were observed. Bacteria penetrate directly between the cells of the root epidermis and remain in intercellular spaces. These empty nodules showed a normal structure with starch accumulation, and they seem to be induced through bacterial signaling at a distance.

The Lotus snf mutants develop normal nitrogen-fixing nodules after inoculation with Mesorhizobium loti. Nodulation is induced through infection threads, and infected cells of nodules are filled with bacteroids, as in wild-type plants. Average nodule numbers are comparable to the wild type inoculated with $M$. loti. Inoculation of snf mutants with $M$. loti does not completely suppress spontaneous nodulation, although the number is drastically reduced. These results demonstrate that mutations present in snf mutants do not compromise normal rhizobial-induced nodulation and suggest at least two alternative mechanisms. Negative regulators may be inactivated or mutated genes could still serve an integrated function in root-nodule development. Nodule numbers in snf mutants decrease under high nitrogen concentration. Nodulation is inhibited at $20 \mathrm{mM}$ nitrate, revealing nitrogen as a main regulator for both rhizobial-induced nodulation and spontaneous nodulation. This is comparable to responses in spontaneously nodulating alfalfa and clover (Blauenfeldt et al. 1994; Schultze and Kondorosi 1998; Truchet et al. 1989). We have shown that spontaneous nodulation in Lotus japonicus is regulated by nitrogen and that spontaneously induced nodule numbers are comparable to rhizobial-induced nodule numbers. We infer that the autoregulatory mechanism involved in regulating number and biomass of rhizobial-induced nodules appears to function in $s n f$ mutants.

Further evidence for a comparable physiological response of spontaneous root nodules and rhizobial-induced nodules were obtained from ethylene inhibition studies. Comparable to previous results with rhizobial-induced nodulation of vetch, Medicago spp. and Lotus japonicus (Heidstra et al. 1997; Nukui et al. 2000; Oldroyd et al. 2001), we have shown that ethylene also regulates spontaneous nodulation negatively by inhibition of nodule development at high concentrations of ethylene precursor ACC. Unexpectedly, the same concentrations allow rhizobial nodulation of $s n f$ mutants as well as wild-type plants. This indicates a strict ethylene regulation of snf nodulation in the absence of rhizobia and an attenuated response upon inoculation with rhizobia. The presence of microsymbionts might influence the response. Alternatively, a different balance of hormones induced in the presence of rhizobia could be involved. We conclude that spontaneous nodulation in Lotus japonicus is regulated by nitrate and ethylene as previously demonstrated for rhizobial-induced nodulation. This further substantiates that snf nodules are genuine root nodules with major physiological response patterns comparable to wild-type nodules. Development of spontaneous nodules in front of the protoxylem poles, where ACC synthase transcripts are less abundant (Heidstra et al. 1997), also suggests a functional ethylene-mediated regulation in snf mutants.

Spontaneous nodulation was earlier thought to be restricted to tetraploid and allogamous species, two features allowing enrichment of the genome for more diversity, as reported in alfalfa and clover (Blauenfeldt et al. 1994; Truchet et al. 1989). Finding the spontaneously nodulating mutants in a diploid species like Lotus japonicus proves that spontaneous nodulation is not specific to tetraploid species and provides an opportunity to understand the mechanisms involved in nodule development by cloning and characterization of genes responsible for the phenotype. Uncoupling plant organogenesis from rhizobial signaling, snf mutants make genetic analysis of plant genes involved in nodule organogenesis a less complex task. This unique class of mutants capable of form- ing spontaneous nodules will, therefore, bring new insights into nodule organogenesis and organ development.

\section{MATERIALS AND METHODS}

\section{Biological material.}

A collection of 2,400 EMS-mutagenized M3 families of Lotus japonicus ecotype Gifu (Perry et al. 2003) was screened for spontaneous nodulation mutants. Each M3 family originates from the mix of seeds from two to over 10 individual M2 plants. The number of seeds screened varies with the number of M2 lines contributing seeds to each M3 family. Approximately 200 seeds were screened per M2 line represented in a M3 family. In total, more than 500,000 individual plants were screened for spontaneous nodules. The snf assignment for spontaneous nodule formation was given to each mutant line, followed by a number according to the order in which they were found. Where more than one mutant plant was found in a pool, they were given the same number and considered as likely siblings. Spontaneous nodulating mutants were found in pools SL467 (snf1), SL1412 (snf2-1), SL1570 (snf2-2), SL679 (snf4), SL642 (snf5), and SL935 (snf6).

\section{Screening procedure and plant growth conditions.}

Seeds were surface-sterilized as described previously (Handberg and Stougaard 1992), were presoaked in sterile water overnight, and were germinated in a double-tray arrangement containing clay granules in the upper tray. The lower tray, containing 3 liters of half concentration of $\mathrm{B}$ and $\mathrm{D}$ nutrients (without $\mathrm{KH}_{2} \mathrm{PO}_{4}$ ) was connected to the upper tray through a wick. Double-trays were covered with foil and autoclaved, and the $\mathrm{KH}_{2} \mathrm{PO}_{4}$ component of $\mathrm{B}$ and $\mathrm{D}$ (Broughton and Dilworth 1971) was added before sowing the seeds. Screening was performed under rhizobia- and nitrogen-free conditions. To assure axenic conditions and avoid contamination with rhizobia, all material and solutions used were either autoclaved or filtersterilized. Plants were grown at $90 \%$ humidity and $25^{\circ} \mathrm{C}$ in isolation for five weeks before screening.

\section{Nodule occupancy assay.}

Spontaneous nodules were excised from each mutant and were surface-sterilized in $12 \%$ hypochlorite, followed by five washes in sterile water. Nodules were crushed individually in 1 $\mathrm{ml}$ of sterile water. Serial dilutions of suspensions were plated on YMA plates and were incubated at $28^{\circ} \mathrm{C}$ for $48 \mathrm{~h}$. Some of the suspension was used to inoculate wild-type seedlings grown on $1 / 4 \mathrm{~B}$ and D media. Rhizobial-induced nodules from wild-type plants were used as a control. Non-surface sterilized spontaneous nodules were crushed directly in $1 \mathrm{ml}$ of sterile water and were used to inoculate wild-type Lotus plants and $100 \mu \mathrm{l}$ of this suspension was plated on YMA plates.

\section{Nitrogen suppression assay.}

Wild-type and snf mutant seeds were surface-sterilized, and seedlings were grown for 5 weeks on solid $1 / 4 \mathrm{~B}$ and D slants with different concentrations of $\mathrm{KNO}_{3}$, ranging from 0 to 20 $\mathrm{mM}$.

\section{Nodulation kinetics.}

Wild-type seedlings and the snf mutants were grown on solid $1 / 4 \mathrm{~B}$ and D slants for 5 weeks. Uninoculated snf mutants were examined at regular intervals for the appearance of spontaneous nodules. To monitor rhizobial nodulation of wild type and snf mutants, seedlings were inoculated with $100 \mu \mathrm{l}$ of a $100 \times$ dilution of a 48-h liquid YMB culture of Mesorhizobium loti NZP2235. Nodule numbers was recorded at the same intervals. 
Ethylene suppression assays.

To assess the effect of ethylene on spontaneous nodulation, snf mutant plants were grown on solid $1 / 4 \mathrm{~B}$ and D slants with different concentrations of ACC or $1.27 \mathrm{nM}$ AVG. The number of nodules developed after rhizobial inoculation of wild-type plants and snf mutants or on snf mutants in the absence of rhizobia, were recorded. At least 40 plants were used for all experiments performed on plates.

\section{Arbuscular mycorrhiza infection test.}

One-week-old seedlings of snf mutants were transferred to chive nurse pots containing clay granules mixed with dried fungal inoculum (Wegel et al. 1998). Seedlings were watered with a nutrient solution (Kosuta et al. 2005) as frequently as needed and were kept in a humid growth cabinet at $25^{\circ} \mathrm{C}$ for 4 to 5 weeks. Plants were scored for mycorrhizal colonization using the ink-staining procedure described by Vierheilig and associates (1998). The screening for mycorrhizal infection was performed in the absence of rhizobia.

\section{Sample preparation and microscopy.}

Nodules from snf mutant plants at 2 and 5 weeks were fixed in $1.5 \%$ paraformaldehyde and $2.5 \%$ glutaraldehyde. The samples are dehydrated by two washes in distilled water and three washes in 2.2 dimethoxypropane solution prior to embedding in Technovit 7100 (according to the manufacturer Haereus Kluwer, Wehrheim, Germany) for longitudinal and transversal sectioning. Thin sections $(4 \mu \mathrm{m})$ were stained with toluidine blue or potassium iodide for light microscopy. A different procedure for staining root tissue was used to investigate early stages (van Spronsen et al. 2001). After assaying for GUS activity, the root tissue was fixed and embedded as described above. After sectioning, the samples were stained with ruthenium red $0.01 \%$ and were prepared for light microscopy.

For electron microscopy, nodules and pieces of nodulated roots were fixed in $2.5 \%$ glutaraldehyde in $0.1 \mathrm{M}$ sodium cacodylate ( $\mathrm{pH} 7.0)$ overnight at $4^{\circ} \mathrm{C}$. The fixed nodules and nodulated roots were then postfixed in $1 \%$ osmium tetroxide, were dehydrated in an ethanol series, and were embedded in Durcupan epoxy resin (Sigma, St. Louis). Ultrathin $(70 \mathrm{~nm})$ sections were taken using a Reichert Ultracut E ultramicrotome. The ultrathin sections were collected on pioloform-coated copper grids and were stained with uranyl acetate $(10 \mathrm{~min})$ and lead citrate $(5 \mathrm{~min})$ before being viewed under a JEOL $1200 \mathrm{EX}$ transmission electron microscope (TEM) (JEOL, Tokyo). The TEM images were collected on phosphor imaging plates (DITABIS Digital Biomedical Imaging Systems AG, Pforzheim, Germany), which were then scanned on a DITABIS micron high resolution imaging plate scanner.

\section{Genetic analysis.}

All snf mutants were selfed, and snf1, snf2-1, snf2-2, and snf4 were crossed to wild-type Lotus japonicus ecotype Miyakojima as described previously (Jiang and Gresshoff 1997). DNA from the F1 leaves was extracted as described by Handberg and Stougaard (1992), and heterozygosity in the F1 plant was confirmed using microsatellite markers showing polymorphisms between Gifu and Miyakojima. Segregating F2 generations were screened for spontaneous nodulation to determine the genetic inheritance. To assess allelism, genetically similar phenotypes were crossed and their F1 phenotypes were observed. For the dominant mutants, the F2 generation was screened for spontaneous nodulation to determine allelism.

\section{Mapping.}

snf1, snf2-1, snf2-2, and snf4 mutants were crossed to Lotus japonicus ecotype Miyakojima as described by Jiang and
Gresshoff (1997). Microsatellite markers showing polymorphism between Miyakojima and Gifu were used to confirm the hybrid nature of the F1 seedlings. F1 plants were selfed, and F2 progenies screened in the absence of rhizobia. Mutant plants showing spontaneous nodules were used to map recessive mutations and wild-type plants were used for dominant mutations. snfl, snf2-1, and snf2-2 were screened 5 weeks postgermination, while the screening for snf4 was performed 7 to 8 weeks postgermination. The linkage maps and mapping technique used to map snf loci were described in Sandal and associates (2005).

\section{Nin and Enod2 expression studies.}

Five-week-old nodules excised from plants grown as described above were harvested. Poly $(\mathrm{A})^{+}$mRNA was extracted from the tissue, using the Dynabeads mRNA direct kit. Treatment of samples, primers used for amplification, and quantitative polymerase chain reaction were described previously (Radutoiu et al. 2003). The whole procedure was performed on three biological replicates for each genotype. Three technical replicates for each biological replicate were included in the light cycler runs.

\section{Analysis of cell division initiation in nodules.}

A snf2-2 mutant was crossed to a transgenic Lotus japonicus line carrying a Nin promoter-GUS reporter gene fusion whose expression marks early nodule cell division. DNA was extracted from leaves of some of the F1 seedlings, and the cross was checked for authenticity, using primers amplifying parts of the GUS coding sequence: 5'-GAGAGGCAGCAAGCATATAC-3' and 5'-CGCGATCCAGACTGAATGCCC-3'. GUSstaining procedure was performed as described previously (Jefferson et al. 1987). Since snf2-2 is dominant, the check for GUS-staining was possible in the F1 generation.

\section{ACKNOWLEDGMENTS}

We thank M. Parniske and T. Wang for making the EMS-mutagenized M3 family seeds available for us. We are grateful to F. Pedersen for plant care. M. Kierans is thanked for assistance in the electron microscopy work. L. Tirichine was supported by the Lotus Training Network grant HPRN-CJ-2000-00086.

\section{LITERATURE CITED}

Allen, E. K., Allen, O. N., and Newman, S. 1953. Pseudonodulation of leguminous plants induced by 2-bromo-3,5 dichlorobenzoic acid. Am. J. Bot. 40:429-435

Asamizu, E., Kato, T., Sato, S., Nakamura, Y., Kaneko, T., and Tabata, S. 2003. Structural analysis of a Lotus japonicus genome. IV. Sequence features and mapping of seventy-three TAC clones which cover the 7.5 $\mathrm{Mb}$ regions of the genome. DNA Res. 10:115-122.

Broughton, W. J., and Dilworth, M. J. 1971. Control of leghemoglobin synthesis in snake beans. Biochem. J. 125:1075-1080.

Blauenfeldt, J., Joshi, P. A., Gresshoff, P. M., and Caetano-Anollés, G. 1994. Nodulation of white clover (Trifolium repens) in the absence of Rhizobium. Protoplasma 179:106-110.

Caetano-Anolles, G., and Gresshoff, P. M. 1991. Plant genetic control of nodulation. Ann. Rev. Microbiol. 45:345-382.

Demont-Caulet, N., Maillet, F., Tailler, D., Jacquinet, J. C., Prome, J. C., Nicolaou, K. C., Truchet, G., Beau, J. M., Denarie, J. 1999. Noduleinducing activity of synthetic Sinorhizobium meliloti nodulation factors and related lipo-chitooligosaccharides on alfalfa. Importance of the acyl chain structure. Plant Physiol. 120:83-92.

Endre, G., Kereszt, A., Kevei, Z., Mihacea, S., Kalo, P., Kiss, G. B. 2002. A receptor kinase gene regulating symbiotic nodule development. Nature 417:962-966.

Finan, T. M., Hirsh, A. M., Leigh, J. A., Johansen, E., Gretchen, A.K., Deegan, S., Walker, G., and Signer, E. R. 1985. Symbiotic mutants of Rhizobium meliloti that uncouple plant from bacterial differentiation. Cell 40:869-877.

Foucher, F., and Kondorosi, E. 2000. Cell cycle regulation in the course of nodule organogenesis in Medicago. Plant Mol. Biol. 43:773-86. 
Fraysse, N., Couder, F., and Poinsot, V. 2003. Surface polysaccharide involvement in establishing the Rhizobium-legume symbiosis. Eur. J. Biochem. 270:1365-1380.

Gage, D. J. 2004. Infection and invasion of roots by symbiotic, nitrogenfixing rhizobia during nodulation of temperate legumes. Microbiol. Mol. Biol. Rev. 68:280-300.

Geurts, R., Fedorova, E., and Bisseling, T. 2005. Nod factor signaling genes and their function in the early stages of Rhizobium infection. Curr. Opin. Plant Biol. 8:346-352.

Goodlass, G., and Smith, K. A. 1979. Effects of ethylene on root extension and nodulation of pea (Pisum sativum L.) and white clover (Trifolium repens L.). Plant Soil 51:387-395.

Gray, J. X., de Maagd, R. A., Rolfe, B. G., Johnston, A. W. B., and Lugtenberg, B. J. J. 1992. The role of the Rhizobium cell surface during symbiosis. Pages 359-376 in: Molecular Signals in Plant-Microbe Communications. D. P. S.Verma, ed. CRC Press, Boca Raton.

Handberg, K., and Stougaard, J. 1992. Lotus japonicus, an autogamous diploid legume species for classical and molecular genetics. Plant J. 2:487-496.

Hayashi, M., Miyahara, A., Sato, S., Kato, T., Yoshikawa, M., Taketa, M., Hayashi, M., Pedrosa, A., Onda, R., Imaizumi-Anraku, H., Bachmair A., Sandal, N., Stougaard, J., Murooka, Y., Tabata, S., Kawasaki, S., Kawaguchi, M., and Harada, K. 2001. Construction of a genetic linkage map of the model legume Lotus japonicus using an intraspecific $\mathrm{F}_{2}$ population. DNA Res. 8:301-310.

Heidstra, R., Yang, W. C., Yalcin, Y., Peck, S., Emons, A., van Kammen, A., and Bisseling, T. 1997. Ethylene provides positional information on cortical cell division but is not involved in Nod factor-induced root hair tip growth in Rhizobium-legume interaction. Development 124:17811787.

Hirsch, A. M., Bhuvaneswari, T. V., Torrey, J. G., and Bisseling, T. 1989. Early nodulin genes are induced in alfalfa root outgrowths elicited by auxin transport inhibitors. Proc. Natl. Acad. Sci. U.S.A. 86:12441248 .

Hirsch, A. M., Mckhann, H. I., and Löber, M. 1992. Bacterial-induced changes in plant form and function. Int. J. Plant Sci. 153:S171-S181.

Hirsch, A. M., and Fang, Y. 1994. Plant hormones and nodulation: What's the connection? Plant Mol Biol. 26:5-9.

Jefferson, R. A., Kavanagh, T. A. and Bevan, M. W. 1987. GUS fusions: $\beta$ glucuronidase as a sensitive and versatile gene fusion marker in higher plants. EMBO (Eur. Mol. Biol. Organ.) J. 6:3901-3907.

Jiang, Q., and Gresshoff, P. M. 1997. Classical and molecular genetics of the model legume Lotus japonicus. Mol. Plant-Microbe Interact. 10:59-68.

Joshi, P. A., Caetano-Anollés, G., Graham, E. T., and Gresshoff, P. M. 1991. Ontogeny and ultrastructure of spontaneous nodules in alfalfa (Medicago sativa). Protoplasma 162:1-11.

Joshi, P. A., Caetano-Anollés, G., Graham, E. T., and Gresshoff, P. M. 1993. Ultrastructure of transfer cells in spontaneous nodules of alfalfa (Medicago sativa). Protoplasma 172:64-76.

Karas, B., Murray, J., Gorzelak, M., Smith, A., Sato, S., Tabata, S., Szczyglowski, K. 2005. Invasion of Lotus japonicus root hairless 1 by Mesorhizobium loti involves the nodulation factor-dependent induction of root hairs. Plant Physiol. 137:1331-1344.

Kato, T., Sato, S., Nakamura, Y., Kaneko, T., Asamizu, E., and Tabata, S. 2003. Structural analysis of a Lotus japonicus genome. V. Sequence features and mapping of sixty-four TAC clones which cover the $6.4 \mathrm{Mb}$ Regions of the genome. DNA Res. 10:277-285.

Kawaguchi, M., Motomura, T., Imaizumi-Anraku, H., Akao, S., and Kawasaki, S. 2001. Providing the basis for genomics in Lotus japonicus: The accessions Miyakojima and Gifu are appropriate crossing partners for genetic analyses. Mol. Genet. Genomics 266:157-66.

Kawaguchi, M., Imaizumi-Anraku, H., Koiwa, H., Niwa, S., Ikuta, A., Syono, K., and Akao, S. 2002. Root, root hair, and symbiotic mutants of the model legume Lotus japonicus. Mol. Plant-Microbe Interact. 15:1726.

Kistner, C., Winzer, T., Pitzschke, A., Mulder, L., Sato, S., Kaneko, T., Tabata, S., Sandal, N., Stougaard, J., Webb, K. J., Szczyglowski, K., and Parniske M. 2005. Seven Lotus japonicus genes required for transcriptional reprogramming of the root during fungal and bacterial symbiosis. Plant Cell 17:2217-2229.

Kosuta, S., Winzer, T. and Parniske M. 2005. Arbuscular mycorrhiza. Pages 87-95 in: Lotus japonicus handbook. A. J. Márquez, ed. Springer, Dordrecht, The Netherlands.

Krusell, L., Madsen, L. H., Sato, S., Aubert, G., Genua, A., Szczyglowski, K., Duc, G., Kaneko, T., Tabata, S., de Bruijn, F., Pajuelo, E., Sandal, N., and Stougaard, J. 2002. Shoot control of root development and nodulation is mediated by a receptor-like kinase. Nature 420:422-426.

Leigh, J. A., Signer, E. R., and Walker, G. C. 1985. Exopolysaccharidedeficient mutants of Rhizobium meliloti that form ineffective nodules.
Proc. Natl. Acad. Sci. U.S.A. 82:6231-6235.

Lerouge, P., Roche, P., Faucher, C., Maillet, F., Truchet, G., Prome, J. C., Denarie, J. 1990. Symbiotic host-specificity of Rhizobium meliloti is determined by a sulphated and acylated glucosamine oligosaccharide signal. Nature 344:781-4.

Libbenga, K. R., and Harkes, P. A. A. 1973. Initial proliferation of cortical cells in the formation of root nodules in Pisum sativum L. Planta 114:17-28.

Limpens, E., Franken, C., Smit, P., Willemse, J., Bisseling, T., and Geurts, R. 2003. LysM domain receptor kinases regulating rhizobial Nod factor-induced infection. Science 24:630-633.

Madsen Bjørn, E., Heegaard Madsen, L., Radutoiu, S., Olbryt, M., Rakwalska, M., Szczyglowski, K., Sato, S., Kaneko, T., Tabata, S. Sandal, N. et al. 2003. A receptor kinase gene of the LysM type is involved in legume perception of rhizobial signals. Nature 425:637-640.

Niwa, S., Kawaguchi, M., Imazumi-Anraku, H., Chechetka, S. A., Ishizaka, M., Ikuta, A., and Kouchi, H. 2001. Responses of a model legume Lotus japonicus to lipochitin oligosaccharide nodulation factors purified from Mesorhizobium loti JRL501. Mol Plant-Microbe Interact. 14:848-56.

Nukui, N., Ezura, H., Yuhashi, K. I., Yasuta, T., and Minamisawa, K. 2000. Effects of ethylene precursor and inhibitors for ethylene biosynthesis and perception on nodulation in Lotus japonicus and Macroptilium atropurpureum. Plant Cell Physiol. 41:893-897.

Oldroyd, G. E., Engstrom, E. M., and Long, S. R. 2001. Ethylene inhibits the Nod factor signal transduction pathway of Medicago truncatula. Plant Cell 13:1835-1849.

Oldroyd, G. E. D., and Downie, A. 2004. Calcium, kinases and nodulation signaling in legumes. Mol. Cell Biol. 5:566-576.

Pawlowski, K., and Bisseling, T. 1996. Rhizobial and actinorrhizal symbioses: What are the shared features? Plant Cell 8:1899-1913.

Pedrosa, A., Sandal, N., Stougaard, J., Schweizer, D., and Bachmair, A. 2002. Chromosomal map of the model legume Lotus japonicus. Genetics 161:1661-1672.

Perry, J. A., Wang, T. L., Welham, T. J., Gardner, S., Pike, J. M., Yoshida S., and Parniske, M. 2003. A TILLING reverse genetics tool and a webaccessible collection of mutants of the legume Lotus japonicus. Plant Physiol. 131:866-871.

Peters, N. K., and Crist-Esters, D. K. 1989. Nodule formation is stimulated by the ethylene inhibitor aminoethoxyvinylglycine. Plant Physiol. 91:690-693.

Radutoiu, S., Heegaard Madsen, L., Bjørn Madsen, E., Felle, H. H., Umehara, Y., Grønlund, M., Sato, S., Nakamura, Y., Tabata, S., Sandal, N., and Stougaard, J. 2003. Plant recognition of symbiotic bacteria requires two LysM receptor-like kinases. Nature 425:585-592.

Sandal, N., Krusell, L., Radutoiu, S., Olbryt, M., Pedrosa, A., Stracke, S., Sato, S., Kato, T., Tabata S., Parniske, M., Bachmair, A., Ketelsen, T., and Stougaard, J. 2002. A genetic linkage map of the model legume Lotus japonicus and strategies for fast mapping of new loci. Genetics 161:1673-1683.

Sandal, N., Rørby Petersen, T., Murray, J., Umehara, Y. Karas B., Yano, K., Kumagai, H., Yoshikawa, M., Saito, K., Hayashi, M., et al. 2005. Genetics of Symbiosis in Lotus japonicus: Recombinant inbred lines, comparative genetic maps and map position of 35 symbiotic loci. Mol. Plant-Microbe Interact. 19:80-91.

Schauser, L., Roussis, A., Stiller, J., and Stougaard, J. 1999. A plant regulator controlling development of symbiotic root nodules. Nature 11:191-195.

Schmidt, J. S., Harper, J. E., Hoffman, T. K., and Bent, A. F. 1999. Regulation of soybean nodulation independent of ethylene signaling. Plant Physiol. 119:951-959.

Schultze, M., and Konodorosi, A. 1998. Regulation of symbiotic root nodule development. Annu. Rev. Genet. 32:33-57.

Searle, I. R., Men, A. E., Laniya, T. S., Buzas, D. M., Iturbe-Ormaetxe, I., Carroll, B. J., Gresshoff, P. M. 2003. Long-distance signaling in nodulation directed by a CLAVATA1-like receptor kinase. Science 299:109112.

Spaink, H. P. 2000. Root nodulation and infection factors produced by rhizobial bacteria. Annu. Rev. Microbiol. 54:257-88.

Sprent, J. I., and EMBRAPA, 1980. Root nodule anatomy, type of export product and evolutionary origin in some Leguminosae. Plant Cell Environ. 3:35-43

Stracke, S., Kistner, C., Yoshida, S., Mulder, L., Sato, S., Kaneko, T., Tabata, S., Sandal, N., Stougaard, J., Szczyglowski, K., and Parniske, M. 2002. A plant receptor-like kinase required for both bacterial and fungal symbiosis. Nature 417:959-962.

Tansengco, M. L., Hayashi, M., Kawaguchi, M., Imaizumi-Anraku, H., and Murooka, Y. 2003. crinkle, a novel symbiotic mutant that affects the infection thread growth and alters the root hair, trichome, and seed development in Lotus japonicus. Plant Physiol. 131:1054-1063. 
Truchet, G., Barker, D. G., Camut, S., de Billy F., Vasse, J., and Huguet, T. 1989. Alfalfa nodulation in the absence of Rhizobium. Mol. Gen. Genet. 219:65-68.

van de Wiel, C., Scheres, B., Franssen, H., van Lierop, M. J., van Lammeren, A., van Kammen, A., and Bisseling, T. 1990. The early nodulin transcript ENOD2 is located in the nodule parenchyma (inner cortex) of pea and soybean root nodules. EMBO (Eur. Mol. Biol. Organ.) J. 9:1-7.

van Spronsen, P. C., Grønlund, M., Pacios Bras, C., Spaink, H. P., and Kijne, J. W. 2001. Cell biological changes of outer cortical root cells in early determinate nodulation. Mol. Plant-Microbe Interact. 14:839-847.
Vierheilig, H., Coughlan, A. P., Wyss, U. R. S., and Piché, Y. 1998. Ink and vinegar, a simple staining technique for arbuscular-mycorrhizal fungi. Appl. Env. Microbiol. 64:5004-5007.

Wegel, E., Schauser, L., Sandal, N., Stougaard, J., and Parniske, M. 1998. Mycorrhiza Mutants of Lotus japonicus define genetically independent steps during symbiotic infection. Mol. Plant-Microbe Interact. 11:933936.

Wopereis, J., Pajuelo, E., Dazzo, F. B., Jiang, Q., Gresshoff, P. M., de Bruijn, F. J., Stougaard, J., and Szczyglowski, K. 2000. Short root mutant of Lotus japonicus with a dramatically altered symbiotic phenotype. Plant J. 23:97-114. 